\title{
Anaphylaktischer Schock nach Injektion von Solu-Decortin H
}

\author{
Anaphylactic Shock After Injection of Solu-Decortin H
}

Autor

Institut
I. Feldmann-Böddeker

Hautklinik (Chefärztin: Dr. med. I. Feldmann-Böddeker), DRK-Krankenhaus Chemnitz-Rabenstein

\section{Bibliografie}

Dol $10.1055 / \mathrm{s}-2007-966900$

Akt Dermatol 2007; 33:

365-367 @ Georg Thieme

Verlag KG Stuttgart · New York ISSN 0340-2541

Korrespondenzadresse

Dr. med. Ingrid FeldmannBöddeker

Hautklinik, DRK-Krankenhaus Chemnitz-Rabenstein

Unritzstraße 23

09117 Chemnitz

feldmann-böddeker.ingrid@

drk-chemnitz.de

\section{Zusammenfassung \\ $\nabla$}

Die Abklärung einer unerwünschten Arzneimittelreaktion ist, wie auch in der vorliegenden Kasuistik, in der Regel eine komplexe Aufgabe. Im vorliegenden Fall wurde ein anaphylaktischer Schock, der während einer Allgemeinanästhesie aufgetreten war, diagnostisch recherchiert.

\section{Einleitung \\ $\nabla$}

Arzneimittelinduzierte Hautveränderungen werden bei 2 bis $4 \%$ aller hospitalisierten Patienten beobachtet. Bei vielen Patienten sind arzneimittelbedingte Hautveränderungen so gering ausgeprägt und flüchtig, dass keine diagnostische Abklärung veranlasst wird. Andererseits werden auch häufig Symptome, die in zeitlichem Zusammenhang mit einer Medikation aufgetreten sind, fälschlicherweise als unerwünschte Arzneimittelreaktion gewertet. Die Abklärung einer unerwünschten Arzneimittelreaktion ist wie auch im vorliegenden Fall eine komplexe Aufgabe. Nicht immer lässt sich ein Kausalzusammenhang zwischen Symptomatik und Arzneimittel herstellen, häufig kann letztendlich nur vermutet werden, welches Medikament die Reaktion ausgelöst hat.

\section{Anamnese}

$\nabla$

Bei einer 28-jährigen Patientin wurde eine Kreuzbandplastik des linken Knies in Allgemeinanästhesie und unter Anwendung eines sogenannten 3:1-Blocks durchgeführt. Während der Allgemeinanästhesie entwickelte die Patientin zunächst ein generalisiertes Erythem sowie eine leicht ausgeprägte Gesichtsschwellung. Hierauf wurden 250 mg Solu-Decortin H intravenös injiziert. Kurze Zeit später entwickelte die Patientin einen drastischen Blutdruckabfall, der als ana- phylaktischer Schock gewertet wurde. Nach Gabe von Akrinor, Suprarenin, Tavegil und Kalzium war die Schocksymptomatik rasch rückläufig. Anhand des Narkoseprotokolls wurde recherchiert, welche Medikation prä- und intraoperativ vor Auftreten der Symptome appliziert worden war, nämlich Propofol, Atropin, Rapifen, Pancuronium, MCP, Metamizol, Cefuroxim, Clexane, Diclofenac und Carbostesin. Anamnestisch war Diclofenac ein halbes Jahr vor diesem operativen Eingriff gut toleriert worden. Ebenso wurde Clexane vor ca. 4 Jahren gut toleriert. Weiterhin wurden während des operativen Eingriffes Latexhandschuhe und andere latexhaltige Materialien eingesetzt.

\section{Allergologische Untersuchungs- befunde \\ $\nabla$}

Spezifisches IgE gegen Latex, Penicilloyl G und V wurden nicht nachgewiesen. Im Epikutantest mit Latex, der Innenseite der verwendeten Latexhandschuhe und sämtlichen verabreichten Medikamenten zeigte sich nach einer Ablesung über 96 Stunden keine Reaktion.

Auch im Pricktest mit zwei kommerziellen Latexextrakten, Propofol, Atropin, Rapifen, Pancuronium und MCP-Tropfen zeigte sich keine Reaktion. Im Intrakutantest mit Rapifen $1: 100$ zeigte sich eine positive, mäßig ausgeprägte Sofortreaktion. Zuvor getestete Kontrollpersonen zeigten bei dieser Konzentration keine Reaktion gegen Rapifen. Im Intrakutantest mit Atropin 1: 100, Pancuronium $1: 100$ und $1: 10$ sowie Propofol $1: 100$ zeigte sich keine Reaktion. Im Pricktest und Intrakutantest mit Carbostesin und zahlreichen anderen Ausweichlokalanästhetika zeigte sich in den Verdünnungen $1: 100$ und $1: 10$ ebenfalls keine Reaktion. Im subkutanen Provokationstest mit den Ausweichlokalanästhetika Ultracain 1\% und Scandicain $1 \%$ zeigte sich jeweils keine Reak- 
tion bis zu einer kumulativen Gesamtdosis von 2,25 ml. Ein Latexhandschuh wurde im Handschuhtragetest gut toleriert. Im Pricktest und Intrakutantest mit Metamizol 1:10 000 und $1: 1000$ sowie Diclofenac $1: 100$ und $1: 10$ sowie weiteren Ausweichanalgetika zeigte sich keine Reaktion. Im oralen Provokationstest wurden die Ausweichanalgetika Paracetamol $500 \mathrm{mg}$ und Ibuprofen $600 \mathrm{mg}$ gut toleriert. Im Pricktest mit Cefuroxim, weiteren Cephalosporinen, Penicillinen und Ausweichantibiotika zeigte sich keine Reaktion, auch nicht im Intrakutantest mit Penicillin G $1: 1000$ und $1: 100$. Im Pricktest und Intrakutantest mit Clexane und weiteren Heparinen $1: 100,1: 10$ und pur zeigte sich keine Reaktion bei einer Ablesung über 7 Tage.

\section{Pricktest mit Solu-Decortin H und weiteren Glukokortikoiden

Keine Reaktion gegen Solu-Decortin H 50 mg (Prednisolon mit Succinat) und Prednabene (Prednisolon ohne Succinat).

Deutlich positive Sofortreaktion gegen Hydrokortison 100 Rotexmedica (Hydrocortison mit Succinat) und gegen Urbason solubile $6 \mathrm{mg}$-Injektionslösung (Methylprednisolon mit Succinat). Bei zahlreichen zuvor in unserem Allergielabor getesteten Patienten war keine Reaktion gegen Hydrocortison 100 Rotexmedica oder Urbason solubile $6 \mathrm{mg}$-Injektionslösung aufgetreten. Keine Reaktion zeigte sich auch gegen Volon A solubile $40 \mathrm{mg}$ Injektionslösung (Triamcinolon ohne Succinat), Fortecortin $8 \mathrm{mg}$ Inject (Dexamethason ohne Succinat) und Celestan solubile $4 \mathrm{mg}$ Injektionslösung (Betamethason ohne Succinat).

\section{Intrakutantest mit Glukokortikoiden \\ $\nabla$}

Deutlich positive Sofortreaktion mit einer Quaddel von $2 \times 2 \mathrm{~cm}$ gegen Solu-Decortin H 50 mg 1: 100 (succinatgebundenes Prednisolon). Zahlreiche zuvor im Allergielabor getestete Patienten hatten keine Reaktion gegen Solu-Decortin H in dieser Verdünnung entwickelt. Keine Reaktion gegen die anderen, bereits im Pricktest nicht reagierenden, nicht succinatgebundenen Kortikoide $1: 100,1: 10$ und pur, inklusive nichtsuccinatgebundenem Prednisolon und Methylprednisolon.

\section{Diskussion \\ $\nabla$}

Während einer Allgemeinanästhesie erlitt die Patientin zunächst ein generalisiertes Erythem und eine Gesichtsschwellung, zusätzlich nach Injektion von $250 \mathrm{mg}$ Solu-Decortin H (succinatgebundenes Prednisolon) einen anaphylaktischen Schock. Symptomauslösend für den anaphylaktischen Schock war nach den anamnestischen Angaben des Narkoseprotokolls und der im Intrakutantest nachgewiesenen, deutlich positiven Soforttypreaktion gegen Solu-Decortin H aller Wahrscheinlichkeit nach das injizierte Solu-Decortin $\mathrm{H}$.

Interessant war weiterhin, dass sich im Intrakutantest mit Prednisolon, das nicht an Succinat gebunden war, keine Reaktion zeigte, und dass sich andererseits auch ausgeprägte Soforttypreaktionen im Pricktest mit succinatgebundenem Methylprednisolon und Hydrokortison zeigten. Von daher besteht bei der Patientin eine Soforttypsensibilisierung offensichtlich nur gegen succinatgebundene Kortikoide. Ein Bezug von reinem Succinat zu einer weiteren allergologischen Testung war leider nicht möglich. In der Literatur wird selten über das Auftreten einer Soforttypallergie gegen succinatgebundene Kortikoide berichtet, eine Soforttypallergie gegen Succinat wurde nach unserer Recherche bislang nicht beschrieben.

Insbesonders nach intravenöser Stoßtherapie mit hohen Dosen von Glukokortikoiden wurden plötzliche Todesfälle beschrieben, ebenso anaphylaktische Reaktionen wie Urtikaria und Bronchospasmus.

Bei bekannter Intoleranz von Acetylsalicylsäure und Asthmatikern werden Soforttypallergien gegen Glukokortikoide besonders häufig beschrieben.

Um der Patientin künftig verträgliche Kortikoide empfehlen zu können, empfahlen wir weiterhin die Durchführung eines oralen und auch intravenösen Provokationstestes mit nichtsuccinatgebundenen Kortikoiden. Zu dieser Testung ist die Patientin leider nicht mehr erschienen.

Beim Intrakutantest mit Rapifen 1:100, das u. a. vor Auftreten des generalisierten Erythems und der Gesichtsschwellung verabreicht worden war, zeigte sich eine mäßig ausgeprägte Soforttypreaktion. Von daher wurde die Eingangssymptomatik vermutlich durch Rapifen ausgelöst. Bedacht werden muss allerdings auch, dass es sich bei dem verabreichten Rapifen, Propofol und auch Pancuronium um histaminliberierende Medikamente handelt.

Die Patientin sollte künftig nicht mehr mit succinatgebundenen Kortikoiden und Rapifen behandelt werden. Vorsichtshalber empfahlen wir auch vor Einleitung einer erneuten Allgemeinanästhesie ein Antihistaminikum zu injizieren. Ein entsprechender Allergiepass wurde der Patientin ausgehändigt.

Bei der Diagnostik von Arzneimittelunverträglichkeiten ist die Erhebung einer ausführlichen Anamnese unerlässlich. Unter anderem müssen sämtliche, in den letzten vier Wochen applizierten Medikamente und deren Zusammensetzung erfasst werden. Bei den allergologischen Hauttestungen sollten sämtliche verabreichte Präparate sowie sofern erhältlich, auch deren Inhaltsstoffe, getestet werden. Wenn möglich sollten auch Reinsubstanzen, Medikamente ähnlicher chemischer Struktur, Medikamente der gleichen Wirkstoffgruppe und Ausweichmedikamente getestet werden. Unter sorgfältiger Nutzen-Risiko-Analyse kann gegebenenfalls auch eine Provokationstestung im Sinne einer Re-Exposition mit dem vermutlich symptomauslösenden Medikament, alternativ auch eine Provokationstestung mit Ausweichmedikamenten erfolgen. Wesentlich ist nach erfolgter Testung die Bestimmung der Kausalität. Zwischen der Reaktion und der Gabe eines Medikamentes kann ein ursächlicher Zusammenhang mit unterschiedlichen Graden der Wahrscheinlichkeit bestehen. Dieser Zusammenhang muss aufgrund medizinisch plausibler Informationen und Überlegungen sowie statistischer Wahrscheinlichkeiten etabliert werden. Der endgültige Beweis, dass ein bestimmtes Arzneimittel eine Nebenwirkung hervorgerufen hat, ist oft schwer zu erbringen. Aufgrund der Vielfältigkeit der klinischen Manifestationen, der oft nicht geklärten Pathogenese und der großen Anzahl auslösender Medikamente, ist die Diagnostik der kutanen Arzneimittelreaktion oft eine Herausforderung. Um künftig schwere Reaktionen zu vermeiden und auch die Arzneimittelsicherheit zu erhöhen, sollten Patienten, die eine vermutliche arzneimittelbedingte Reaktion erlitten haben, unbedingt einer allergologischen Diagnostik und Beratung zugeführt werden. 


\section{Anaphylactic Shock After Injection of Solu-Decortin H} $\nabla$

The diagnosis of adverse drug reactions is mostly a difficult job. In the reported case an anaphylactic shock was investigated which occurred during a general anaesthesia.

\section{Literatur}

1 Kurzhals G et al. Anaphylaktische Reaktion auf Succinatester von Kortikosteroiden. Allergologie 1995; 18: 156 - 158

2 Alexion $C$ et al. Klinische Bedeutung allergologischer Reaktionen bei Glukokortikoidbehandlung. Laryngo-Rhino-Otol 1999; 78: 573 - 578

$3 \mathrm{Kamm} \mathrm{GL,} \mathrm{Hagmeyer} \mathrm{K.} \mathrm{Allergic-type} \mathrm{reactions} \mathrm{to} \mathrm{corticosteroids.} \mathrm{Ann}$ Pharmacother 1999; 33: 451-460

4 Barbaud $A$ et al. Guidelines for performing skin tests with drugs in the investigation of cutaneous adverse drug reactions. Contact dermatitis 2001; 45: $321-328$

5 Bircher A. Arzneimittelallergie und Haut. Stuttgart: Georg Thieme Verlag, 1996: 40-41, 127-153

6 Jäger L, Merk M. Arzneimittelallergie. Jena: Gustav Fischer Verlag, 1996: $99-101,166-178$

7 Bork K. Arzneimittelnebenwirkungen an der Haut. Stuttgart, New York: Schattauer Verlag, 1998: 371 - 378

8 Ammon MPT. Arzneimittelneben- und Wechselwirkungen. Stuttgart: Wissenschaftliche Verlagsgesellschaft mbH, 2001: 1099-1100

9 Burgdorff T, Venemalm L, Vogt T, Landthaler M, Stolz W. IgE-mediated anaphylactic reaction induced by succinate ester of methylprednisolone. Annals of Allergy, Asthma and Immunology 2002; 89: 425 - 428

10 Fulcher DA, Katelanis C. Anaphylactoid reaction to intravenous hydrocortisone sodium succinate: a case report and literature review. Med J Aust 1991; 154: 783-784

11 Nakamura $\mathrm{H}$, Matsusett $\mathrm{H}$ et al. Clinical evaluation of anaphylactic reactions to intravenous corticosteroids in adult asthmatics. Respiration Vol 2002; 69: 309-313
Buchbesprechung

Manual der Wundheilung

Chirurgisch-dermatologischer Leitfaden der modernen Wundbehandlung

Wild T, Auböck J (Hrsg)

Wien: Springer, 2007. 354 S., 200 Abb., davon 80 in Farbe.

Geb. $98.00 €$

ISBN-13: 978-3-211-25212-3

Dr. Thomas Wild (Universitätsklinik für Chirurgie, Wien) und Prim. Univ.-Prof. Dr. Josef Auböck (Abteilung für Dermatologie und Venerologie, AKH Linz) haben als Herausgeber mit Unterstützung internationaler Autoren einen Leitfaden zur modernen Wundbehandlung geschrieben.

In 33 Kapiteln werden basale Zusammenhänge zum aktuellen Stand der Wundbehandlung dargestellt. Es werden zunächst Modelle der verbalen Wunddokumentation und digitalen Wundanalyse aufgezeigt und auf die pathophysiologischen Grundlagen der Wundheilung eingegangen. In einzelnen Kapiteln werden verschiedene chronische Wunden wie z. B. diabetischer Fuß, Unterschenkelgeschwüre, Dekubitalulzera, Verbrennungen und Osteomyelitis mit ihren diagnostischen und differenzialdiagnostischen Überlegungen erörtert. Eindrucksvolle Fotos zeigt das Kapitel von N. Sepp (Universitätsklinik Dermatologie und Venerologie, Innsbruck) zu ulzerösen Hauterkrankungen als Differenzialdiagnose zu chronischen Wunden. Einem breiten Spektrum an therapeutischen Möglichkeiten zur Wundbehandlung wird Raum gegeben. Dies sind neben den modernen Wundauflagen die physikalischen und biologischen (Fliegenlarven) Therapiemöglichkeiten, das Tissue Engineering bis hin zu kinesiologischen Verfahren. In drei Kapiteln wird die Vakuumtherapie erläutert und diskutiert. Auch die Bedeutung der Ernährung für die Prophylaxe und Therapie von Wundheilungsstörungen wird ausführlich erwähnt. Interessante Beiträge finden sich am Ende des Buches zu Fragen der Ökonomie und Arzthaftung in der Wundbehandlung. Der Anhang besteht aus vier Artikeln zum Management von Wundinfektionen mit kritischen Beurteilungen zu topischen antimikrobiellen Therapien und praktischen Therapieempfehlungen für den klinisch tätigen Arzt.

Die einzelnen Kapitel sind klar strukturiert und durch umfangreiches Bild- und Tabellenmaterial aufgelockert. Sie beginnen mit einer farblich abgehobenen Zusammenfassung und enden mit Prüfungsfragen und oftmals auch interessanten Hinweisen für die Praxis. Die Literaturstellen sind jedem Kapitel angehängt.

Dieser Leitfaden behandelt sehr umfangreich alle Aspekte der modernen Wundbehandlung und ist für jeden dermato-chirurgisch tätigen Arzt eine große Hilfe sowohl im Verständnis als auch in der Behandlung von chronischen Wunden.

Sabine Bethge, Wiesbaden 